\title{
Fundamental Relationships in Galactic Disks
}

\author{
Stuart D. Ryder \\ School of Physics, University of New South Wales, Sydney, NSW 2052, Australia \\ Present address: Joint Astronomy Centre, \\ 660 N. A'Ohoku Place, Hilo, HI 96720, USA \\ sryder@jach.hawaii.edu \\ Received 1996 August 8, accepted 1996 November 8
}

\begin{abstract}
Although a number of correlations have been demonstrated between observable parameters in galaxies, such as surface brightness, luminosity, metallicity, etc., debate continues as to which of these parameters are truly fundamental. Following a major surface photometry program and $\mathrm{H}_{\mathrm{II}}$ region abundance analysis, we have been able to show that the surface density of recent massive star formation, the surface density of stars already formed, and the mean oxygen abundance at a given galactic radius are all fundamentally related within and between the disks of spiral galaxies. Such relationships can serve as powerful constraints on models of galactic evolution, requiring, for instance, a star formation law dependent not only on gas surface density, but also on the total mass surface density.
\end{abstract}

Keywords: galaxies: evolution — galaxies: fundamental parameters - galaxies: spiral

\section{Introduction}

Fundamental relationships between observable parameters in astrophysical objects can serve as powerful tools for helping us interpret the universe around us. One of the most familiar examples of such a relationship is the Hertzsprung-Russell diagram, which relates a star's luminosity with its temperature (as determined from its colour/spectral type). Once it was realised that the HR diagram charted a star's evolutionary path, astronomers were able to use it to derive other fundamental quantities, such as the ages and distances of star clusters.

The search for a simple galactic analogue of the HR diagram has not been quite so fruitful. For elliptical galaxies, the central velocity dispersion, the effective radius (or the total luminosity), and the mean surface brightness are all related, and define what is commonly referred to as the 'Fundamental Plane' (Djorgovski \& Davis 1987; Jørgensen, Franx \& Kjærgaard 1996). For dwarf galaxies, correlations have been claimed between surface brightness and luminosity, colour and luminosity, metallicity and luminosity, and thus between metallicity and surface brightness, but it is not yet clear which of these (if any) is the truly fundamental correlation, and which may be just a selection effect (Edmunds \& Phillipps 1989). In the case of spiral galaxies, initial hopes that the Hubble type of a galaxy would prove to be tightly correlated with other parameters have not panned out. There is of course the Tully-Fisher relation between a spiral galaxy's luminosity and its maximum rotational velocity. However, in view of the unknown role played by dark matter in maintaining this rotational velocity at large radii, the TF relation is really only of limited use in analysing the evolution of spiral galaxies.

Assuming that such a fundamental relationship exists and can be found for spiral galaxies, it would:

- Argue for a common origin, and provide clues to the formation process.

- Serve as an empirical endpoint for galaxy evolution models, and thereby help to constrain the plethora of star formation prescriptions, initial mass functions, gas exchange processes, etc.

- Provide a useful (relative) distance indicator.

- Define truly abnormal galaxies as those that depart significantly from the mean trend.

It is worth pointing out that all of the relationships just mentioned involve global properties of the galaxy. This paper describes the search for correlations of local properties, both within and between the disks of spiral galaxies. Much of this work is described in more detail in Ryder \& Dopita (1994; hereafter Paper I) and in Ryder (1995; hereafter Paper II).

\section{A Relationship between Stellar Surface Density and Star Formation Rate}

Our search for new fundamental relationships in galactic disks began with a major imaging study of 34 nearby $\left(V_{\text {hel }}<2000 \mathrm{~km} \mathrm{~s}^{-1}\right)$ southern spiral galaxies in both the $\mathrm{V}$ and I bands (the I band being more sensitive to the older, low-mass stellar population) as well as in the $\mathrm{H} \alpha$ line (which highlights the $\mathrm{H}$ II regions ionised by newly formed massive stars, and thus can be used as a measure of the current star formation rate; Kennicutt 1983). Surface photometry using the GASP package was then carried out to determine 

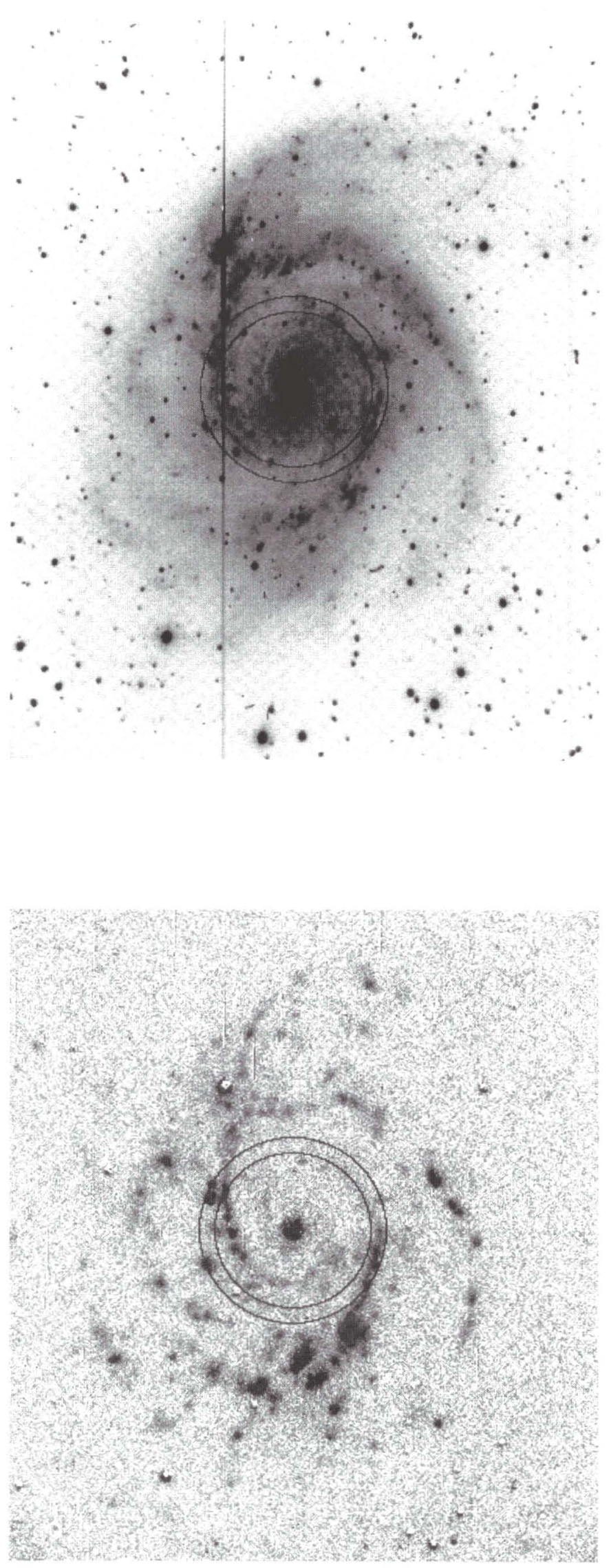

Figure 1-Images of the nearby spiral galaxy NGC 2997 in the V-band (top) and H $\alpha$ line (bottom) from the MSSSO 40 inch telescope. The concentric circles in each image mark annular zones of equal radii, within which the mean surface brightnesses are to be compared. 
the disk orientation parameters, and thus the radial variation of deprojected surface brightness (assuming the disk to be optically thin throughout) in each band. We then asked the question: Is there any correlation between the azimuthally averaged stellar surface brightness at a given radius in a galaxy (as marked in the upper panel of Figure 1), and the azimuthally averaged star formation rate (lower panel) at that same radius? In this way, we are effectively normalising by the galaxy's scale length, without the need for complex bulge-to-disk decompositions.

As Figure 6 of Paper I shows, there is indeed a very pronounced trend, amongst all the galaxies in our sample, in the way that the $\mathrm{H} \alpha$ surface brightness decreases with decreasing I-band surface brightness, with the main departures from perfectly uniform behaviour being that some loci lie slightly above (fainter in $\mathrm{H} \alpha$ at all points) or slightly below (brighter in $\mathrm{H} \alpha$ ). In order to investigate whether there may be a 'second parameter' at work, we have plotted the size of this offset against various global galactic parameters. A good linear correlation was found with the logarithm of the total $\mathrm{H}$ I gas content per unit area of the optical disk (Figure 2), in the sense that it is the more gas-rich spirals that appear brighter in $\mathrm{H} \alpha$ for a given I-band surface brightness than the mean. This is perhaps to be expected, on the basis that galaxies with low gas surface densities will not be capable of forming stars at the same level as those with higher gas densities. Figure 3 shows just how well the loci agree after the offset correction is applied.

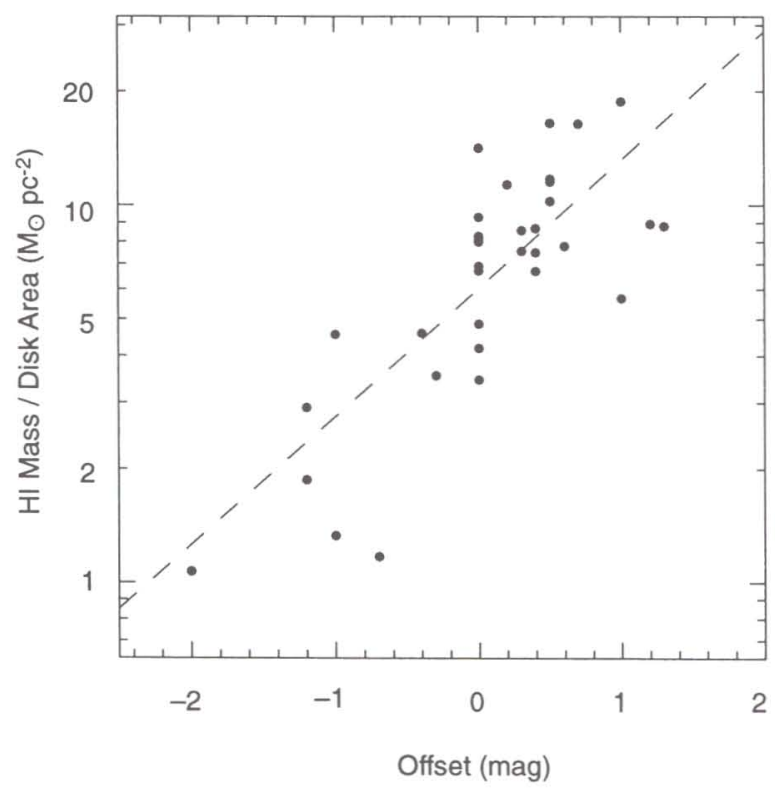

Figure 2-Plot of the total $\mathrm{H}$ I gas content normalised by the area of the optical disk, on a logarithmic scale, against the offset (in magnitudes per square arcsecond) needing to be applied to the $\mathrm{H} \alpha$ surface brightnesses in order to bring them into agreement with the mean locus of $\mathrm{H} \alpha$ versus I-band surface brightness. The dashed line is a linear least-squares fit to the points.

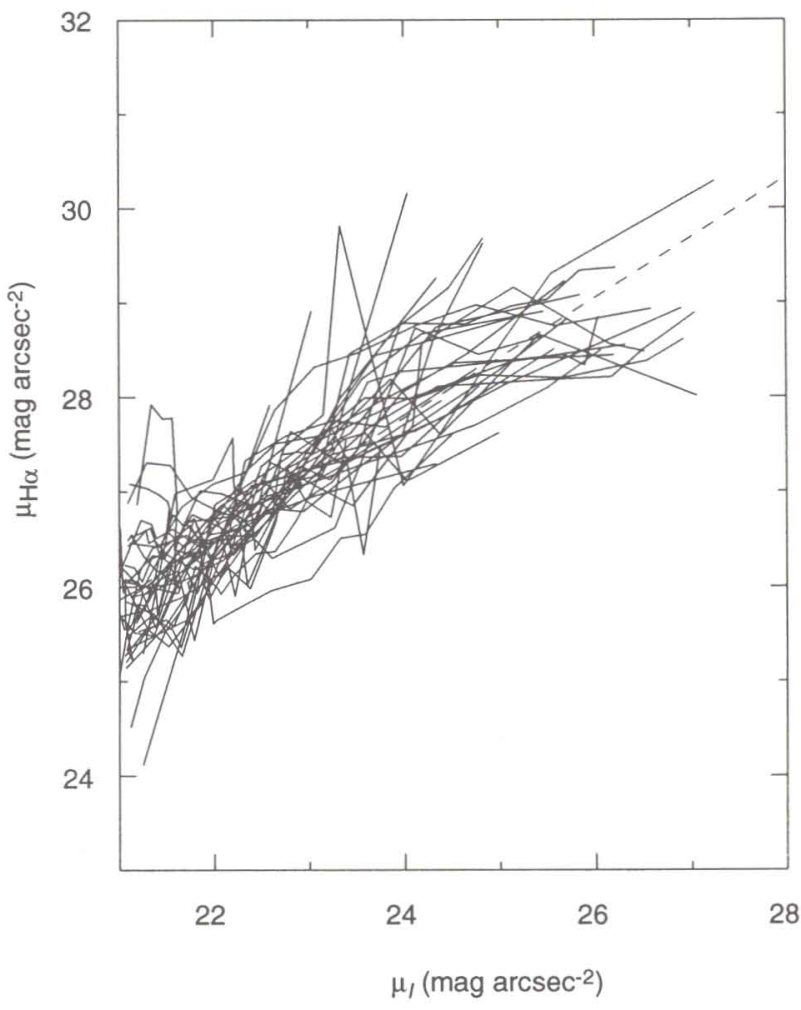

Figure 3-Individual loci of $\mathrm{H} \alpha$ surface brightness versus I-band surface brightness for the 34 spiral galaxies in our sample, after the offsets from Figure 2 are applied, compared with the mean trend (dashed line).

\section{Modelling the Observed Relationship}

Before we can make use of this new relationship to constrain models, we must first convert from observable quantities (surface brightnesses) to the sorts of parameters (stellar surface density, star formation rate) tracked by the models. To get from surface luminosity density to surface mass density of stars, we make use of the finding by Buchhorn (1992) that the mass-to-light ratio in the I-band (for Sc galaxies at least) has a value of $4 \cdot 2 \pm 1 \cdot 3$. Similarly, by combining the calculations of Wilson (1983) for the production rate of UV photons as a function of stellar lifetime and mass with the standard Case $\mathrm{B}$ recombination in $\mathrm{H}$ II regions, it can be shown that a surface luminosity density (extinction-corrected) of $1 \mathrm{~L}_{\odot} \mathrm{pc}^{-2}$ in $\mathrm{H} \alpha$ equates to a formation rate for massive $\left(M>10 \mathrm{M}_{\odot}\right)$ stars of $3 \mathrm{M}_{\odot} \mathrm{pc}^{-2} \mathrm{Gyr}^{-1}$; using the initial mass function (IMF) of Kennicutt (1983) to extrapolate over all masses $\left(0 \cdot 1-100 \mathrm{M}_{\odot}\right)$ gives a total star formation rate of $23 \mathrm{M}_{\odot} \mathrm{pc}^{-2} \mathrm{Gyr}^{-1}$. Although there have been suggestions that the stellar IMF may vary with age, metallicity, etc., no systematic trends have been identified (Gilmore 1989), and we therefore assume a constant IMF in order to minimise the number of free parameters in the model.

Our model traces the evolution of a series of radial 'zones' having asymptotic total mass surface 


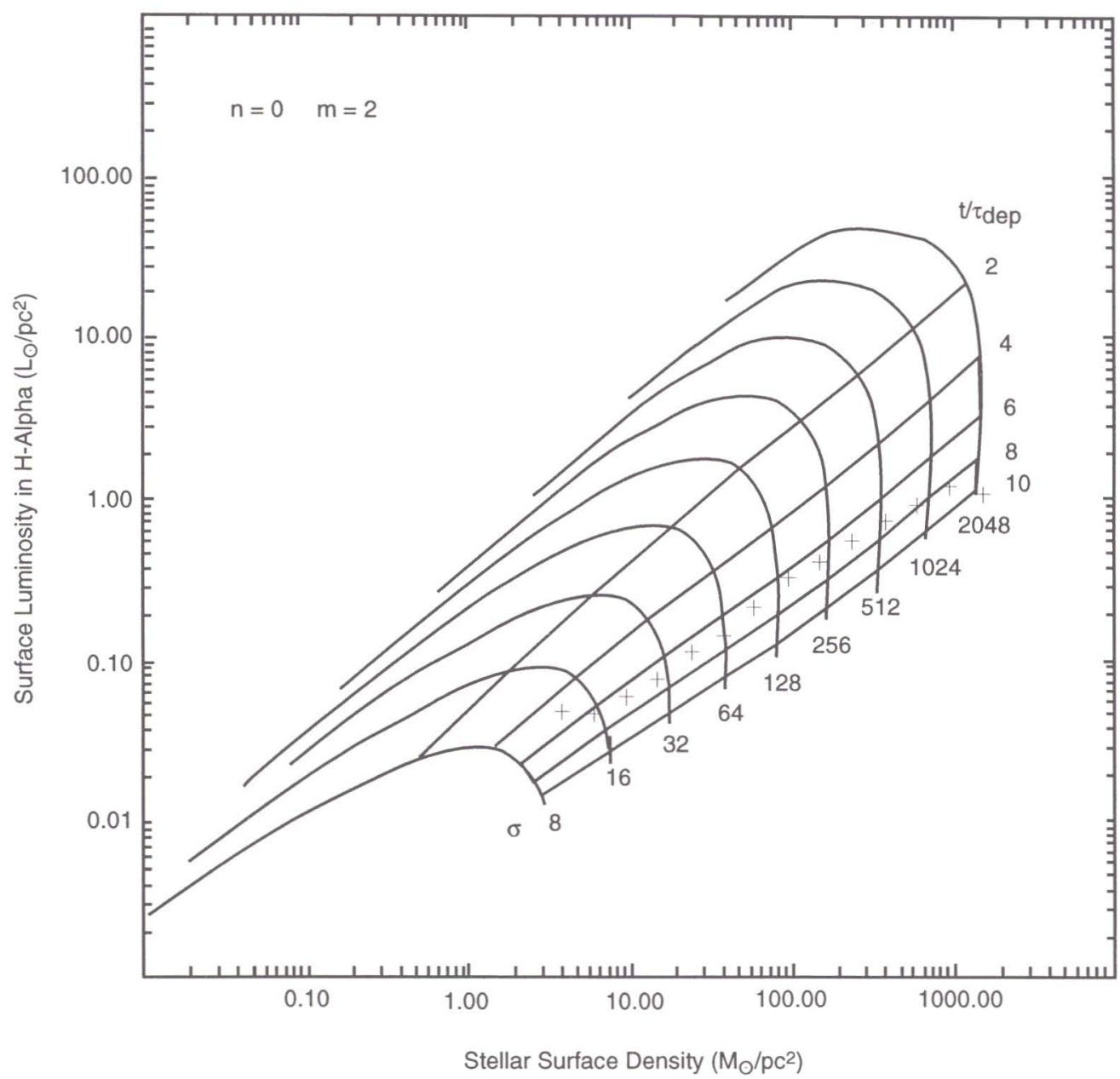

Figure 4-Evolution of our model galactic disk, overlaid on the calibrated observational relationship between star formation rate and stellar surface density (crosses), for the 'classical' Schmidt Law with a second-order dependence on gas surface density. Evolutionary tracks are shown for each mass zone, as well as isochrones, expressed in terms of the gas depletion timescale (Dopita \& Ryder 1994).

densities of $8,16,32, \cdots, 2048 \mathrm{M}_{\odot} \mathrm{pc}^{-2}$. No mass exchange between these zones is allowed. Gas is assumed to fall into these zones at an exponentially decreasing rate. Following the suggestion of Dopita $(1985,1990)$ that the star formation rate may be a function of both the total and the gas surface densities, we adopt a 'compound' Schmidt-type law for star formation:

$$
\frac{\mathrm{d} \sigma_{\star}}{\mathrm{d} t}=\epsilon \sigma_{\mathrm{tot}}^{n} \sigma_{\mathrm{g}}^{m} .
$$

For $n=0$, this defaults to the 'classical' Schmidt Law. The efficiency parameter $\epsilon$ is chosen to give solar neighbourhood conditions $\left(\sigma_{\text {tot }}=67 \mathrm{M}_{\odot} \mathrm{pc}^{-2}\right.$, $\sigma_{\mathrm{g}}=13 \cdot 5 \mathrm{M}_{\odot} \mathrm{pc}^{-2}$ ) after $13 \mathrm{Gyr}$ of evolution.

The resulting evolutionary tracks for each zone, as well as isochrones, are shown superimposed on our calibrated observational correlation in Figure 4 for the case of $n=0, m=2$. By running a series of models with varying combinations of $n$ and $m$, we were able to constrain their sum to $1 \cdot 5<(n+m)<2 \cdot 5$, but cannot rule out particular values of the indices on the basis of the star formation rate/stellar surface brightness relationship alone.

\section{A Relationship between Stellar Surface Density and Oxygen Abundance}

Returning to Figure 1, we now ask the question: Is there any correlation between the azimuthally averaged stellar surface brightness at a given radius in a galaxy, and the abundance of oxygen within the $\mathrm{H}$ II regions at that same radius? Such a relationship has been touched upon by McCall (1982), Webster \& Smith (1983), Wevers (1984), Edmunds \& Pagel (1984) and, more recently, by VilaCostas \& Edmunds (1992), but never satisfactorily explained. The oxygen abundance can be determined spectroscopically to an accuracy $\sim 0.2$ dex using the empirical calibration of $12+\log (\mathrm{O} / \mathrm{H})$ versus $R_{23}$, the sum of the $\left[\mathrm{O}_{\mathrm{II}}\right] \lambda \lambda 3726,3729$ and $\left[\mathrm{O}_{\mathrm{III}}\right] \lambda \lambda 4959,5007$ line strengths normalised to $\mathrm{H} \beta$ (Edmunds \& Pagel 1984; Dopita \& Evans 1986).

The oxygen abundances in $97 \mathrm{H}$ II regions for six of the galaxies in our sample have been measured, 


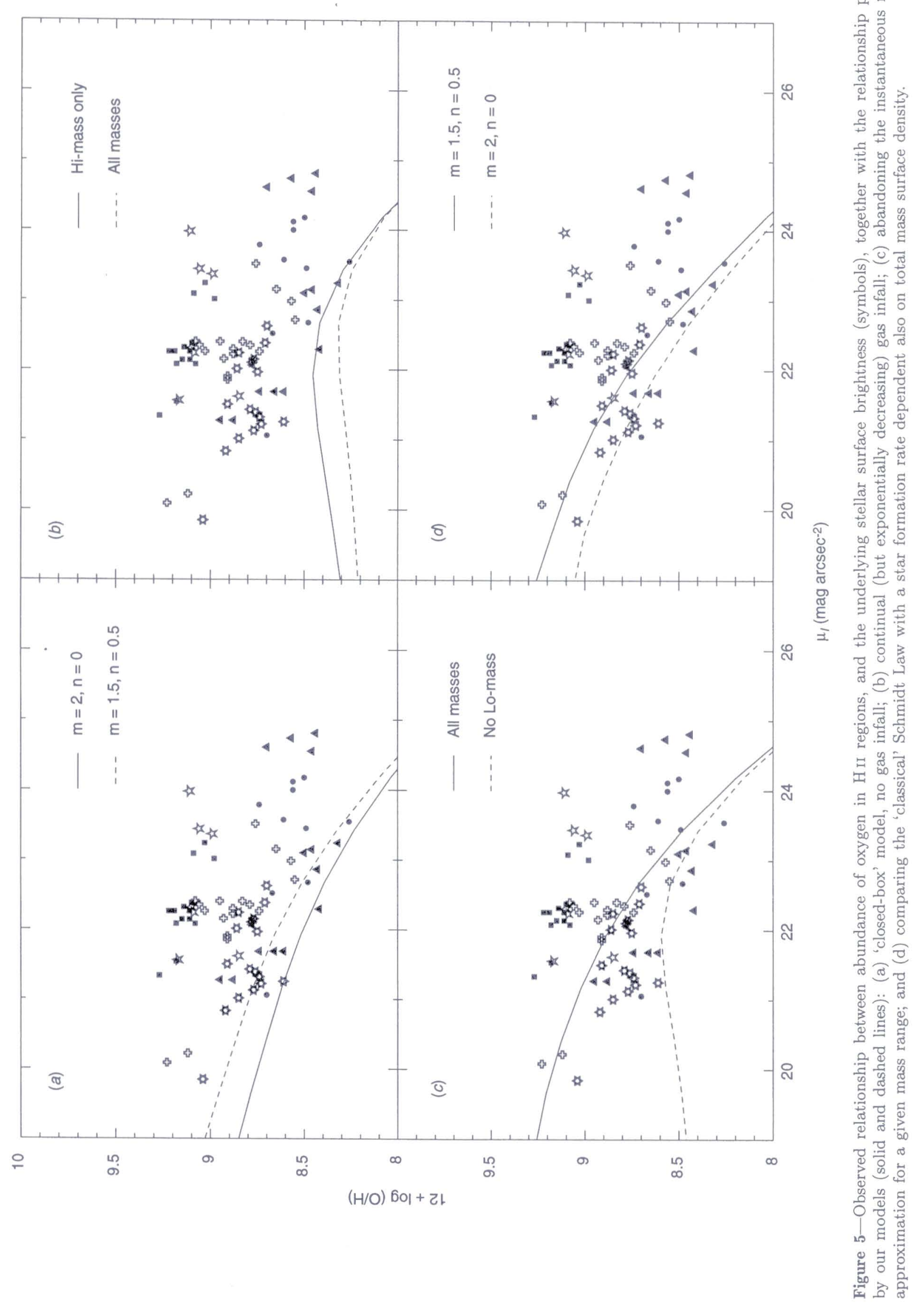


and plotted against the underlying stellar surface brightness in Figure 5. This plot reconfirms the existence of a local metallicity/surface brightness relationship in galactic disks, and offers us a second constraint on the above models.

Our first model uses the closed-box approximation (no gas infall, each zone starts with its full quota of gas) and instantaneous recycling approximation (high-mass stars die immediately, returning some enriched gas to the ISM; low-mass stars lock away all of their gas forever). As Figure 5a shows, this simple model follows almost the same slope as the observed metallicity/surface brightness relationship, but the oxygen yields are too low. Boosting the amount of oxygen present, by enhancing high-mass star formation at the expense of low-mass stars, would, however, violate the stellar surface brightness/star formation rate relationship.

A more realistic model with gas infall has an even more serious problem (Figure 5b); the dilution of enriched gas in higher-density regions by the continual infall of pristine gas. This problem disappears, however, when one abandons the instantaneous recycling approximation (Figure $5 \mathrm{c}$ ), and takes full account numerically of stellar lifetimes, and the delayed return of oxygen to the ISM by low-mass stars forming planetary nebulae late in the galaxy's lifetime. Although the amount of oxygen released by each star is small, the sheer number of low-mass stars formed in each episode of star formation (relative to high-mass stars) means that they could make a significant contribution to the gas-phase oxygen abundance at late epochs.

Finally, having identified the most realistic model involving the minimum number of assumptions, we have again experimented with various combinations of the total and gas surface density indices $n$ and $m$ (Figure $5 \mathrm{~d}$ ). This time, we see that the 'classical' Schmidt Law with $m=2$ falls somewhat short of satisfying the metallicity/surface brightness relationship, while the model that includes a mild dependence on the total surface mass density does slightly better. The fit could conceivably be improved further by allowing for some variations in the $M / L_{\mathrm{I}}$ ratio or in the IMF. Phillipps \& Edmunds (1991) also found that self-regulating star formation models that included some dependence on the total mass surface density (e.g. Dopita 1985; Matteucci et al. 1989) did a much better job of matching the observed metallicity/surface brightness/density relationships than did basic Schmidt Laws in a closed-box model. Thus there is good evidence that a star formation law dependent on both the total and the gaseous surface densities does somewhat better than a classical Schmidt Law at simultaneously satisfying both of the relationships presented here.

\section{Conclusions}

We have demonstrated that the following three (azimuthally averaged) observational parameters are correlated at each radius in the disks of nearby spiral galaxies:

(1) $\sigma_{I}$ - the surface density of old stars;

(2) $\dot{\sigma}_{\star}$ - the recent formation rate of massive stars per unit area; and

(3) $\log (\mathrm{O} / \mathrm{H})$ the mean gas-phase abundance of oxygen in $\mathrm{H}$ II regions, the current sites of star formation,

such that

$$
\dot{\sigma}_{\star} \propto\left(\sigma_{\mathrm{I}}\right)^{\frac{2}{3}}\left(\bar{\sigma}_{\mathrm{HI}}\right)
$$

and

$$
\mathrm{O} / \mathrm{H} \propto\left(\sigma_{\mathrm{I}}\right)^{\frac{1}{4}}
$$

where $\bar{\sigma}_{\mathrm{HI}}$ is the $\mathrm{HI}$ mass per unit area of the star-forming disk. As a consequence of these new observational relationships, and our attempts to interpret them using galactic evolution models, two important results have emerged:

(1) The return of oxygen to the ISM by dying low-mass stars late in the galaxy's lifetime can make a significant contribution to the gas-phase oxygen abundance at late epochs.

(2) The 'classical' Schmidt Law for star formation cannot simultaneously satisfy both the $\left(\sigma_{\mathrm{I}}, \dot{\sigma}_{\star}\right)$ and the $\left(\sigma_{\mathrm{I}},(\mathrm{O} / \mathrm{H})\right)$ relationships; some dependence on the total mass surface density (or some other quantity that scales with it) is also required.

It should be emphasised that these results have only been shown to hold in the disks of spiral galaxies; they break down in the bulge region (perhaps due to the different dynamical environment there), and thus may not necessarily lead to a global relationship. While we may never come up with a fundamental relationship for spiral galaxies as conceptually simple as the Hertzsprung-Russell diagram for stars, the relationships presented here could well form the basis of a 'Fundamental Plane' for galactic disks.

\section{Acknowledgments}

SDR acknowledges the receipt of a UNSW ViceChancellor's Postdoctoral Research Fellowship, and useful discussions with M. Dopita, M. Bureau, and S. Driver.

Buchhorn, M. 1992, Ph.D. Thesis, Australian National University

Djorgovski, S., \& Davis, M. 1987, ApJ, 313, 59

Dopita, M. A. 1985, ApJ, 295, L5

Dopita, M. A. 1990, in The Interstellar Medium in External Galaxies, ed. H. A. Thronson \& J. M. Shull (Dordrecht: Kluwer), 437

Dopita, M. A., \& Evans, I. N. 1986, ApJ, 307, 431

Dopita, M. A., \& Ryder, S. D. 1994, ApJ, 430, 163

Edmunds, M. G., \& Pagel, B. E. J. 1984, MNRAS, 211, 507 
Edmunds, M. G., \& Phillipps, S. 1989, MNRAS, 241, 9P Gilmore, G. 1989, in The Milky Way as a Galaxy (19th Advanced Course of the Swiss Society of Astronomy and Astrophysics), ed. R. Buser \& I. King (Sauverny: Geneva Observatory), 281

Jørgensen, I., Franx, M., \& Kjærgaard, P. 1996, MNRAS, 280,167

Kennicutt, R. C. 1983, ApJ, 272, 54

McCall, M. L. 1982, PhD thesis, University of Texas

Matteucci, F., Franco, J., François, P., \& Treyer, M. A. 1989, Rev. Mexicana Astron. Astrofis., 18, 145
Phillipps, S., \& Edmunds, M. G. 1991, MNRAS, 251, 84

Ryder, S. D. 1995, ApJ, 444, 610

Ryder, S. D., \& Dopita, M. A. 1994, ApJ, 430, 142

Vila-Costas, M. B., \& Edmunds, M. G. 1992, MNRAS, 259, 121

Webster, B. L., \& Smith, M. G. 1983, MNRAS, 204, 743

Wevers, B. M. H. R. 1984, PhD thesis, University of Groningen

Wilson, I. R. G. 1983, PhD thesis, Australian National University 